\title{
Using Automated Text Processing to Assess the Patient Experience of an On-Demand Tele-Urgent Care
}

\author{
Saif KHAIRAT ${ }^{\mathrm{a}, 1}$, Xinyi ZHANG ${ }^{\mathrm{a}}$, Makena BOYD ${ }^{\mathrm{b}}$, Barbara EDSON ${ }^{\mathrm{b}}$ and Robert \\ GIANFORCARO ${ }^{\mathrm{b}}$ \\ ${ }^{a}$ University of North Carolina at Chapel Hill, Chapel Hill, NC, USA \\ ${ }^{\mathrm{b}}$ UNC Health - Chapel Hill, NC, USA
}

\begin{abstract}
Novel methods are needed to evaluate the perceptions of patients using telehealth. Automated text processing methods presents a golden opportunity to classify and analyze unstructured survey responses from patients. This study analyzed 585 unstructured entries from telehealth patients. Satisfied patients who returned for a second visit applauded the efficiency and physician interactions. While unsatisfied patients who did not return for a second visit complained of misdiagnosis and inefficiencies in e-prescription. Patient experience was significantly different between weekdays and weekends $(\mathrm{p}<0.05)$. Overall, teleurgent are convenient for patients however, there are current facilitators related to patient-provider interaction and health information exchange that need further optimization.
\end{abstract}

Keywords. Telemedicine, Patient, Experience

\section{Introduction}

The use of patient surveys for care is one of the most effective ways that providers can measure patient safety and satisfaction. While the typical use of Likert scale questions provides valuable feedback for providers and health care administrators, the free text parts of these patient surveys are often underutilized (1). The information within these free text comments on the quality of care provide context in which the patient scored different aspects of care on the more structured questions of the survey. Given high the variation in patient comments on surveys, the use of word clouds and natural language processing (NLP) can be used to automatically identify themes of care. Automatic analysis of free text can be used to identify themes in patient tweets after their visit with to the hospital (2).

As more people are exposed to virtual care, the more opportunity there is to learn how to improve current operations to better suit patients. It is paramount to understand the nuances and experiences of patients using a virtual care system to improve the overall quality of care. The goal of this study was to evaluate the patient experience within a tele-urgent care center using automated text analysis of patient survey comments.

\footnotetext{
${ }^{1}$ Corresponding Author, Saif Khairat, PhD, MPH, University of North Carolina at Chapel Hill, 428 Carrington Hall, Chapel Hill, NC 27514 USA; E-mail: saif@unc.edu.
} 


\section{Methods}

A Southeastern Medical Center in the U.S. utilized telehealth to provide on-demand care to individuals using board-certified physicians. The 24-hour virtual urgent care service was developed to aid individuals with medical needs who may need urgent care and need help when walk-in clinics are closed or because they have limited independent mobility. The online, on-demand service is available to the public regardless of their demographics.

After the tele-urgent encounter, patients received a satisfaction survey about their telemedicine experience including a question about their overall satisfaction (measured by 5-point likert scale) and open text comments about the patient's experience. We measured satisfaction as According to the outcomes above, we created 2 new variables. One is a binary variable with two levels: Satisfied/Dissatisfied and it is based on the overall experience of the encounter. If the overall experience is Excellent/Very good/ Good/ Fair, the value of this variable would be "Satisfied". If the overall experience is Poor, the value of it would be "Dissatisfied". The other is also a binary variable with two levels: Revisit and NotRevisit. The level of revisit means after the first encounter, the patients re-used the service at least once. The level of NotRevisit means after the first encounter, the patients never revisited. Institutional Review Board approval was obtained. Data was collected between 2018-2019 through the web-portal and imported into a secure, HIPAA compliant business intelligence server. The data was reviewed and cleaned by two graduate students. We applied the ordinal logistic regression function named polr from $\mathrm{R}$ (version 3.5.1) package polr to determine if there is a significant relationship between overall experience and the day of the week (weekday/weekend).

First, we analyzed the comments made by all patients during the first encounter to detect the reason why patients revisited or never revisited after their first visit. First, we split these comments into 4 different groups: (1) comments from patients who revisited and were satisfied with the first encounter, and (2) comments from patients who were unsatisfied with their first encounter and never revisited. In order to find the reasons why patients revisited or never revisited, we analyzed the patient comments and summarized the keywords of comments across different patient groups based on word frequencies using package nltk and wordcloud in Python (version 3.6.5). The outcome of this study was the perceptions of patients who revisited or never revisited after their first encounter.

Finally, we used word clouds to visualize the keywords that the comments are about in each group. In the plots, terms that appeared more frequently in the cleaned text would stand out. In addition, we provided a frequency table of common words in each group using function CountVectorizer. By comparing keywords across different groups, we can conclude what people liked or disliked about the virtual service in general.

\section{Results}

A total of 585 survey responses were analyzed from 546 unique patients, of which 400 (73\%) were female, and $207(38 \%)$ patients were in 35-49 age group. Of the 602 encounters, 431(73.7\%) encounters were completed on weekday, and 154 (26.3\%) were on weekend. The percentage of poor overall experience on weekday encounters was significantly higher than weekend encounters, figure 1. Excellent patient satisfaction levels were significantly higher on weekends (71\%) compared to weekdays $(63 \%)$ (pvalue $<0.05)$. Poor ratings of the service on the weekdays $(8 \%)$ were double the ratings on weekends (4\%). An association was found between the volume of visits and patient 
satisfaction levels. Weekdays had substantially higher visit volume compared to the weekends and patient satisfaction was lower on weekdays compared to the weekends.

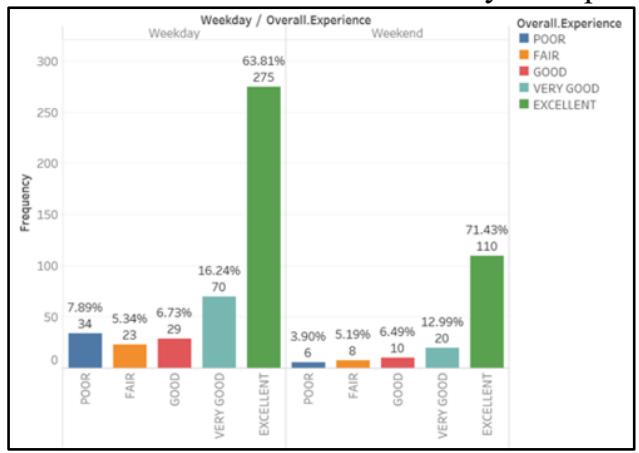

Figure 1. Patient satisfaction on the weekdays versus weekends

There were 37 patients who were satisfied with their first visit and revisited later, we noticed that $41 \%$ patients adopted the keyword "excellent". The cohort mainly cared about physician and time in terms of encounter. The keywords like "convenient", "time" and "quick home" are all related to the online convenience property of virtual service, Figure 1. Examples of patients' responses who were satisfied after their first encounter and revisited for a second visit included "how quickly the md returns your call", "I didn't have to take time off of work", and "it was quick and easy".

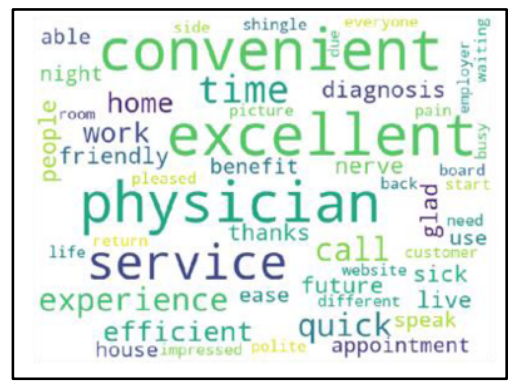

Figure 2. NLP-based word cloud of the perceptions of satisfied patients who revisited for a second visit.

There were 31 unsatisfied patients who did not return, Figure 3. Feedback from these patients included "I have waited almost 24 hours for my daughter's prescription to be called in", "repeatedly waiting for multiple calls and consultations", "Physician offered no advice", and "did not agree with diagnosis".

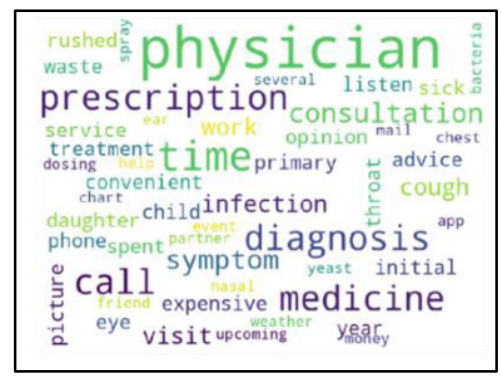

Figure 3. NLP-based word cloud of the perceptions of unsatisfied patients who did not revisit. 


\section{Discussion}

We found that weekdays and video visits had lower patient experience ratings compared to weekends and phone visits. Patients who returned for a second visit had high satisfaction in their first visit. Factors that drove high satisfaction included convenience, not missing work, and excellent recommendations from the treating physician.

Patients who had high satisfaction in their first visit but did not return for a second visit reported that short wait times, pleasant physician interaction, and ease of receiving prescription as drivers of high satisfaction. We observed that patients who did not return for a second visit were mainly unsatisfied with their physician interaction, misdiagnoses, long wait times, and the duration of the visit or feeling rushed during the visit. The findings support our previous analysis of patient satisfaction after using telehealth (3-5).

Patient experience in the telehealth space may be influenced by various factors such as gender, the telehealth modality used, and the specialty of the visit (6). Similarly, provider telehealth competencies play an important role in how patients evaluate their telehealth experience such that telehealth visits that are short or the patient cannot clearly see the provider have lower satisfaction scores.

This study had limitations. We assumed that the reason for some patients not returning for a second visit was due to a satisfaction-related issue, which may not be true in many cases. Although it may be true, patient's health condition may be a confounding factor such that some patients may not have been sick again and thus, did not need a second visit. Also, we did not account for providers' perception of using tele-urgent care to compliment patient views.

In conclusion, tele-urgent care clinics may be beneficial and convenient to patients however, this study reveals that there are certain patient concerns around the quality of patient-provider interaction and privacy concerns that require further enhancements. Patients reported significantly higher satisfaction on weekends compared to weekdays. Satisfied patients who returned for a second visit applauded the efficiency and physician interactions. While unsatisfied patients who did not return for a second visit complained of misdiagnosis and inefficiencies in e-prescription.

\section{References}

[1] Doing-Harris K, Mowery DL, Daniels C, Chapman WW, Conway M. Understanding patient satisfaction with received healthcare services: A natural language processing approach. AMIA Annu Symp Proc. 2016;2016:524-33. Epub 2017/03/09. PMID: 28269848.

[2] Greaves F, Laverty A, Ramirez-Cano D, Pulman S, Moilanen K, Darzi A, et al. Analysis Of Patients' Comments About Hospitals In The English Nhs Via Twitter, And Comparison With Patient Surveys. Bmj Quality Safety. 2014;23(4):348. doi: 10.1136/bmjqs-2014-002893.5.

[3] Khairat S, Tirtanadi K, Ottmar P, Gudhe R, Austin CA. Would Geriatric Patients Accept Using a Telemedicine Platform for Post ICU-Discharge Follow-Up Visits? Stud Health Technol Inform. 2019;264:1233-7. Epub 2019/08/24. doi: 10.3233/shti190423. PMID: 31438122.

[4] Newlin T, McCall T, Ottmar P, Welch B, Khairat S. Assessing the Satisfaction of Citizens Using Teleconsent in Clinical Research. Stud Health Technol Inform. 2018;247:685-9. Epub 2018/04/22. PMID: 29678048.

[5] Khairat S, Haithcoat T, Liu S, Zaman T, Edson B, Gianforcaro R, et al. Advancing health equity and access using telemedicine: a geospatial assessment. J Am Med Inform Assoc. 2019. Epub 2019/07/25. doi: 10.1093/jamia/ocz108. PMID: 31340022.

[6] Khairat S, Pillai M, Edson B, Gianforcaro R. Evaluating the Telehealth Experience of Patients With COVID-19 Symptoms: Recommendations on Best Practices. Journal of Patient Experience. 2020:2374373520952975. doi: 10.1177/2374373520952975. 